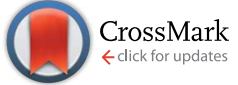

Cite this: RSC Adv., 2015, 5, 19715

Received 15th December 2014 Accepted 11th February 2015

DOI: $10.1039 / c 4 r a 16411 c$

www.rsc.org/advances

\section{Injectable in situ forming hydrogels based on natural and synthetic polymers for potential application in cartilage repair}

\author{
Calogero Fiorica, ${ }^{a}$ Fabio Salvatore Palumbo, ${ }^{a}$ Giovanna Pitarresi, ${ }^{\text {*abc }}$ \\ Alessandro Gulino, 'Stefano Agnello ${ }^{a}$ and Gaetano Giammona ${ }^{\text {ac }}$
}

\begin{abstract}
In this work we prepared two new hyaluronic acid (HA) based in situ forming hydrogels for the potential treatment of articular cartilage damages. In particular the amino derivative of HA (HA-EDA) and its graft copolymer with $\alpha$-elastin (HA-EDA-g- $\alpha$-elastin) were crosslinked, in mild physiological conditions via Michael-type addition, with $\alpha, \beta$-poly( $N$-2-hydroxyethyl)-DL-aspartamide (PHEA) derivatized with divinylsulfone (DV). The swelling and degradation profile of the obtained hydrogels as well as the metabolic activity of incorporated bovine articular chondrocytes were investigated. Histological analysis and scanning electron microscopy (SEM) were performed to analyze the morphology of cells after scheduled times of incubation. Moreover the influence of the $\alpha$-elastin on the elastic modulus of cell free and cell containing hydrogels has been studied through atomic force microscopy (AFM).
\end{abstract}

\section{Introduction}

Cartilage diseases such as osteoarthritis (OA) affect each years world wide thousand of people of all ages and in particular people older than $60 .^{1} \mathrm{OA}$ is a progressive musculoskeletal disorder characterized by gradual degeneration of cartilage that causes serious tissue lesions that can induce pain, immobility and joint destruction. ${ }^{2,3}$ The main characteristic of $\mathrm{OA}$ is an imbalance between chondrocyte anabolic (synthesis) and catabolic (resorptive) activities. ${ }^{4}$ During the course of the disease, the loss of proteoglycans from the extracellular matrix and the disruption of the collagenous fibrillar network lead to the development of lesions that can gradually affect the entire cartilaginous structure. ${ }^{5}$ Cartilage regeneration is particularly challenging because of the poor auto-regenerative properties of the tissue and the peculiar mechanical forces acting in the regeneration site. These issues represent the main reason of the failure of "early tissue engineering" treatments like the grafting of autologous osteochondral tissue or the transplantation of autologous chondrocyte suspensions, introduced to allow or enhance the repair of articular cartilage. ${ }^{5,6}$ Highly porous

${ }^{a}$ Dipartimento di Scienze e Tecnologie Biologiche Chimiche e Farmaceutiche, Sezione di Chimica e Tecnologie Farmaceutiche, Universitá degli Studi di Palermo, Via Archirafi 32, 90123, Palermo, Italy. E-mail: giovanna.pitarresi@unipa.it

${ }^{b}$ IBIM-CNR, Via Ugo La Malfa 153, 90146 Palermo, Italy

'Institute of Biophysics at Palermo, Italian National Research Council, Via Ugo La Malfa 153, 90146 Palermo, Italy

${ }^{d}$ Department of Health Science, Human Pathology Section, School of Medicine, Via del Vespro 129, 90127, Palermo, Italy

$\dagger$ Electronic supplementary information (ESI) available. See DOI: 10.1039/c4ra16411c scaffolds that interact with cells, bioactive molecules and mechanical signals in a dynamic and synergistic manner may contribute to the process of regeneration and could be used to maintain differentiated cells in a given area, or to encourage proliferation of chondrocytes. ${ }^{7,8}$ The development of biomaterials with biological, mechanical and physicochemical properties similar to those of the native extracellular matrix in the cartilage tissue has been the goal of several research groups. Among different types of materials, hydrogels seem to be optimal scaffolds for cartilage regeneration. Indeed, Chung and Burdick report that hydrogels support the transport of nutrients and waste, and they can homogenously hold cells in a $3 \mathrm{D}$ environment, where encapsulated cells typically retain a rounded morphology that may induce a chondrocytic phenotype. Hydrogels are also capable of transducing mechanical loads to exert controlled forces on encapsulated cells, similar to physiological conditions. ${ }^{9}$ Many efforts have been also made to develop systems that can be easily implanted into the body through minimally invasive surgical procedures designed to prevent actions that could further jeopardize the situation of the pathological tissue. In order to obtain injectable hydrogels able to gel in situ, several chemical and physical crosslinking methods, such as photopolymerization, ${ }^{\mathbf{1 0}, 11}$ Schiff-base formation, ${ }^{12}$ horseradish peroxidase (HRP)-mediated chemical crosslinking, Michael-type addition reactions, ${ }^{\mathbf{1 3 , 1 4}}$ and $\alpha$-stacking interactions ${ }^{15}$ have been employed to obtain injectable hydrogels that gel in situ. ${ }^{\mathbf{1 6}}$ The choice of a specific technique derives from the compromise between the characteristics of resulting scaffold and the effect on cell viability. We have already demonstrated that Michael-type addition is a promising way to obtain hydrogels in situ in the presence of cells since this 
reaction could occur in mild conditions in aqueous environment without the need of catalysts or other activants ${ }^{17}$ that could affect cell viability. In this work we have employed an ethylenediamino (EDA) derivative of hyaluronic acid (HA) and $\alpha$-elastin named HA-EDA- $g$ - $\alpha$-elastin to develop an in situ forming hydrogel via Michael-type addition with PHEA-DV, i.e. a synthetic water soluble polymer obtained by functionalizing $\alpha, \beta$-poly( $N$-2-hydroxyethyl)-DL-aspartamide (PHEA) with divinylsulfone (DV). In addition, as a comparison, a second in situ forming hydrogel has been also produced by using the amino derivative of HA (HA-EDA) (without elastin) and PHEA-DV.

PHEA has been employed in the past years as a starting polymer for the development of biomaterials both for tissue engineering and drug delivery purposes..$^{18-22}$ In a previous paper we have demonstrated that the presence of $\alpha$-elastin in HA-EDA$g$ - $\alpha$-elastin based scaffolds increases cell adhesiveness towards rat dermal fibroblasts. ${ }^{23}$ Moreover Nesic et al. reported that protein-based natural polymers may contain ligands that can be recognized by cell-surface. ${ }^{4}$ Swelling behaviour, chemical and enzymatic degradation, together with the possibility to incorporate viable bovine articular chondrocytes have been investigated to verify the possibility of using these hydrogels as injectable scaffolds for cartilage regeneration. Moreover mechanical properties of the obtained hydrogels, alone or in the presence of cells, have been investigated through atomic force microscopy (AFM).

\section{Experimental section}

\subsection{Chemicals}

All reagents were of analytical grade, unless otherwise stated. Bis(4-nitrophenyl) carbonate (4-NPBC), divinyl sulfone (DV), triethylamine (TEA), tetrabutylammonium hydroxide (TBA-OH), Dulbeccos Modified Eagles Medium (DMEM), Dulbeccos phosphate buffered saline (DPBS), trypsin-EDTA solution, amphotericin B solution, penicillin-streptomycin, fetal bovine serum (FBS), hyaluronidase (HAase) from bovine testes were obtained from Sigma-Aldrich (Milano, Italy). Ethylenediamine (EDA), anhydrous dimethylsulfoxide (DMSO), anhydrous dimethylformamide (DMF) were all from Fluka (Milano, Italy). CellTiter 96 AQueous One Solution Cell Proliferation Assay (MTS) was purchased by Promega. $\alpha, \beta$-Poly( $N$-2-hydroxyethyl)DL-aspartamide (PHEA) was prepared and purified according to a procedure reported elsewhere. ${ }^{24}$ PHEA weight-average molecular weight was $41 \mathrm{kDa}\left(M_{\mathrm{w}} / M_{\mathrm{n}}=1.78\right)$ determined by size exclusion chromatography (SEC). Hyaluronic acid (HA) having a low weight-average molecular weight $(230 \mathrm{kDa}$; polydispersity index 1.9) was prepared by acidic degradation as already reported by $\mathrm{Shu}^{25}$ starting from a biotechnological HA sodium salt with a $M_{\mathrm{w}}$ of $1500 \mathrm{kDa}$ (Altergon, Italia). The tetrabutylammonium salt of HA (HA-TBA) was produced as described by Palumbo et $a l^{26}$ The extraction of $\alpha$-elastin from elastin of bovine neck was performed following the procedure reported by Partridge et al. ${ }^{27}$ Briefly $1 \mathrm{~g}$ of bovine elastin was suspended in $0.25 \mathrm{M}$ oxalic acid solution and hydrolyzed by sequential cycles of heating for $1 \mathrm{~h}$ in a water boiling bath. The supernatant was collected by centrifugation and the precipitated solid was treated until complete hydrolysis. The collected soluble fractions were dialyzed against water using a dialysis membrane tube of $3500 \mathrm{Da}$, then freeze-dried. The obtained powder was dissolved in acetic acid solution $0.01 \mathrm{M}$ containing $\mathrm{NaCl} 0.1 \mathrm{M}$ ( $1 \mathrm{~g}$ of hydrolyzed elastin in $10 \mathrm{~mL}$ of acidic solution) and heated at $50{ }^{\circ} \mathrm{C}$ for $15 \mathrm{~min}$ to obtain the $\alpha$-elastin coacervation. The coacervate was collected by centrifugation. The procedure was repeated until no more elastin precipitated. The $\alpha$-elastin was finally dissolved in acetic acid solution $0.1 \mathrm{M}$ and dialyzed against acetic acid solution using a dialysis membrane of 3500 $\mathrm{Da}$ at $4{ }^{\circ} \mathrm{C}$ for 1 day. Finally, the solution was freeze-dried and $\alpha$-elastin was stored as a powder.

\subsection{Apparatus}

The ${ }^{1} \mathrm{H}$-NMR spectra were recorded in $\mathrm{D}_{2} \mathrm{O}$ (Aldrich) using a Bruker Avance II 300 spectrometer operating at $300 \mathrm{MHz}$. Scanning electron microscopy (SEM) images were recorded by using a scanning electron microscope (ESEM QUANTA FEI $200 \mathrm{~F}$ ) with an accelerating voltage of $15 \mathrm{kV}$. Samples were dehydrated by hexamethyldisilazane (Sigma-Aldrich) and dried under vacuum prior to be gold sputtered and analyzed. A Thermo Labsystems Multiskan Ex 96 well microplate photometer was used to evaluate cell viability after MTS test. AFM analysis was conducted by using a Bruker FastScan atomic force microscope equipped with a probe having a spherical $\mathrm{SiO}_{2}$ tip with radius of $3.31 \mu \mathrm{m}$ and a cantilever of $450 \mu \mathrm{m}$ with a constant force of $0.3 \mathrm{~N} \mathrm{~m}^{-1}$ (calculated after calibration with mica surface) (Sqube). Samples of hystologycal analysis were observed with a Leica DMD108 optical digital microscope.

\subsection{Synthesis of ethylendiamino derivative of hyaluronic acid (HA-EDA) and ethylendiamino derivative of hyaluronic acid $\boldsymbol{g}$ - $\alpha$-elastin (HA-EDA- $\boldsymbol{g}$ - $\alpha$-elastin)}

First HA-EDA-TBA was synthesized as reported in our previous work..$^{15}$ Briefly, $500 \mathrm{mg}$ of HA-TBA was dissolved in $45 \mathrm{~mL}$ of anhydrous DMSO then $184 \mathrm{mg}$ of 4-NPBC, dissolved in $3 \mathrm{~mL}$ of DMSO, was added dropwise to the HA-TBA solution at $40{ }^{\circ} \mathrm{C}$ (molar ratio between 4-NPBC and HA-TBA equal to 0.75). The solution was left at the same temperature for $4 \mathrm{~h}$. After this time, $404 \mu \mathrm{L}$ of EDA (molar ratio equal to 10 respect to the moles of 4-NPBC) was added and the solution was left at $40^{\circ} \mathrm{C}$ for $3 \mathrm{~h}$. The obtained HA-EDA-TBA derivative was precipitated in an excess of diethyl ether then washed 4 times in acetone and dried under vacuum. The yield for this reaction was $75 \%$ in weight (respect to the HA-TBA initial weight). To obtain HA-EDA, TBA was eliminated from the final product dissolving the freezedried HA-EDA-TBA in water at a concentration of $1 \%$ in the presence of $500 \mu \mathrm{L}$ of $\mathrm{NaCl}$ saturated aqueous solution. The obtained solution was dialyzed against $\mathrm{NaCl} 5 \%(\mathrm{w} / \mathrm{v})$ solution for 2 days and then against water for other 2 days and the product was recovered by freeze drying. ${ }^{1} \mathrm{H}$ NMR spectrum of HA-EDA derivative in $\mathrm{D}_{2} \mathrm{O}$ showed principal peaks at $\delta 1.9(-\mathrm{NH}-$ $\mathrm{CO}-\mathrm{CH}_{3}$ of $\mathrm{HA}$ ), $\delta 3.1$ (-CO-NH-CH$-\mathrm{CH}_{2}-\mathrm{NH}_{2}$ of $\left.\mathrm{EDA}\right)$, $\delta 3.3 / 3.8$ (pyranosyl $\mathrm{CH}$ of $\mathrm{HA}$ ). The derivatization degree in terms of moles of EDA linked to HA, calculated by ${ }^{1} \mathrm{H}-\mathrm{NMR}$ analysis (spectrum not shown), was $60 \pm 5$ mol\%. HA-EDA- $g$ - 
$\alpha$-elastin copolymer was synthesized according to a synthetic protocol already published. ${ }^{23}$ Briefly $200 \mathrm{mg}$ of $\alpha$-elastin was dissolved in $5 \mathrm{~mL}$ of DMSO, then $35 \mathrm{mg}$ of 4 -NPBC were added and the reaction was maintained at $40{ }^{\circ} \mathrm{C}$ for $4 \mathrm{~h}$. Solution of $\alpha$-elastin activated with 4 -NPBC was added to $25 \mathrm{~mL}$ of anhydrous DMSO containing $550 \mathrm{mg}$ of HA-EDA-TBA derivative. After $3 \mathrm{~h}$ at $40{ }^{\circ} \mathrm{C}$, the reaction was stopped by adding $1 \mathrm{~mL}$ of aqueous $\mathrm{NaCl}$ saturated solution, then precipitated with an excess of acetone (to remove TBA). Samples were washed with acetone-water $8: 2 \mathrm{v} / \mathrm{v}, \mathrm{DMSO}$ and then acetone alone. Obtained products were dried under vacuum, dissolved in water, dialyzed against water (dialysis tubing of 12 000/14 000 cutoff) and finally freeze-dried. The yield for this reaction was $85 \%$ in weight (respect to the HA-EDA initial weight). The amount of $\alpha$-elastin grafted to HA-EDA, calculated by UV determination at $280 \mathrm{~nm}$, was $40 \% \mathrm{w} / \mathrm{w}$ (spectrum not shown).

\subsection{Synthesis of divinylsulfone derivative of $\alpha, \beta$-poly $(\mathrm{N}-2$ - hydroxyethyl)-DL-aspartamide (PHEA-DV)}

An amount of $500 \mathrm{mg}$ of PHEA was dissolved in $10 \mathrm{~mL}$ of anhydrous DMF in the presence of TEA and then DV was added to the solution. The molar ratio between TEA or DV and PHEA repetitive units was set equal to 5 . The reaction was carried out for $24 \mathrm{~h}$ at $50{ }^{\circ} \mathrm{C}$, the product was recovered by precipitation in diethyl ether, purified by at least 5 washings in the same solvent and recovered by under vacuum drying. ${ }^{1} \mathrm{H}-\mathrm{NMR}$ in $\mathrm{D}_{2} \mathrm{O}$ showed peaks at $\delta 2.7$ (m, 2H,-CH- $\left.\mathrm{CH}_{2}-\mathrm{CO}-\mathrm{NH}-\right), \delta 3.2(\mathrm{~m}, 2 \mathrm{H},-\mathrm{NH}-$ $\left.\mathrm{CH}_{2}-\mathrm{CH}_{2}-\mathrm{OH}\right), 3.6\left(\mathrm{~m}, 2 \mathrm{H},-\mathrm{NH}-\mathrm{CH}_{2}-\mathrm{CH}_{2}-\mathrm{OH}\right)$ of PHEA and $\delta 6.3$ and $\delta 6.8\left(3 \mathrm{H}, 2 \mathrm{q}: \mathrm{CH}_{2}=\mathrm{CH}-\right)$ of DV. PHEA-DV weight average molecular weight was measured by SEC analysis with the same conditions already described for PHEA based on PEO/PEG standards. ${ }^{24}$

\subsection{Hydrogel formation and gelation time}

HA-EDA or HA-EDA- $g$ - $\alpha$-elastin were dissolved in DMEM (without other supplement) at a concentration of $3 \% \mathrm{w} / \mathrm{v}$ while PHEA-DV was dissolved in the same solvent at the concentration of $6 \% \mathrm{w} / \mathrm{v}$. To allow the crosslinking reaction, solution of PHEA-DV was added to that of HA-EDA or HA-EDA- $g$ - $\alpha$-elastin and the resultant solution was incubated at $37^{\circ} \mathrm{C}$ under gentle shaking. The weight ratio between HA-EDA or HA-EDA- $g$ - $\alpha$ elastin and PHEA-DV was set to $1: 1$, the final concentration of HA-EDA or HA-EDA- $g$ - $\alpha$-elastin in the gelling solution was $2 \% \mathrm{w} / \mathrm{v}$. The gelation time was qualitatively monitored by the inversion tube test. ${ }^{28}$

\subsection{Swelling and degradation studies}

Swelling data were obtained by comparing the weights of freshly prepared hydrogels with those of the same samples after scheduled times (from 1 to 30 days) of incubation in DPBS $\mathrm{pH}$ 7.4 at $37^{\circ} \mathrm{C}$ in orbital shaker at $80 \mathrm{rpm}$. To obtain the swollen weights, the excess of DPBS from each sample was accurately removed with blotting paper. The swelling percentage was calculated as:

$$
\begin{aligned}
\text { Swelling } \%= & {[(\text { final wet weight }- \text { initial wet weight }) /} \\
& \text { initial wet weight }] \times 100
\end{aligned}
$$

Each experiment was performed in triplicate. For hydrolytic degradation studies, hydrogels were washed at least five times with double distilled water then freeze dried and accurately weighed. Dried hydrogels were submerged into $4 \mathrm{~mL}$ of DPBS pH 7.4 and incubated at $37{ }^{\circ} \mathrm{C}$ in orbital shaker at $80 \mathrm{rpm}$. At scheduled times (from 3 to 30 days), hydrogels were washed five times with double distilled water, freeze dried and weighed. The degradation was expressed as \% of recovered weight, calculated as:

Recovered weight $\%=($ final weight $/$ initial weight $) \times 100$

Enzymatic degradation experiments were performed in the same conditions used for hydrolytic degradation study but each hydrogel was incubated until 72 hours days in DPBD pH 7.4 in the presence of HAase at a concentration of $30 \mathrm{U} \mathrm{mL}^{-1}$. Also enzymatic degradation was expressed as \% of recovered weight. Both hydrolytic and enzymatic degradation experiments were performed in triplicate.

\subsection{Incorporation, metabolic activity evaluation and morphological analysis of bovine articular chondrocytes}

Bovine articular chondrocytes were isolated as reported in a previous published work. ${ }^{29}$ HA-EDA and HA-EDA- $g$ - $\alpha$-elastin were sterilized as powders by UV irradiation for 1 hour using a 125 Watt UV-lamp. A solution of PHEA-DV in DMEM was sterilized by filtrating through a $0.22 \mu \mathrm{m}$ nylon filter. To produce engineered constructs by incorporating cells into the hydrogels, chondrocytes were harvested, resuspended in an appropriate volume of solution of HA-EDA or HA-EDA- $g$ - $\alpha$-elastin in DMEM. The concentration of each HA derivative in the cell containing solution was $3 \% \mathrm{w} / \mathrm{v}$. Afterwards, an appropriate amount of PHEA-DV sterile DMEM solution, at a concentration of $6 \% \mathrm{w} / \mathrm{v}$, was added. 300 microliters of the so obtained gelling system containing $5 \times 10^{5}$ cells was poured into each well of a 48 well plate and incubated at $37^{\circ} \mathrm{C}$ in $\mathrm{CO}_{2} 5 \%$ atmosphere for $30 \mathrm{~min}$ to allow the crosslinking reaction. $500 \mu \mathrm{L}$ of complete DMEM, obtained by supplementing DMEM with $10 \% \mathrm{v} / \mathrm{v}$ of FBS, $1 \% \mathrm{v} / \mathrm{v}$ of penicillin-streptomycin solution, $1 \% \mathrm{v} / \mathrm{v}$ of glutamine solution and $0.1 \% \mathrm{v} / \mathrm{v}$ amphotericin B solution, was then added to each hydrogel. The weight ratio between HA-EDA or HA-EDA- $g$ $\alpha$-elastin and PHEA-DV was set equal to $1: 1$ and the final concentration of each HA derivative in the gel forming solutions was $2 \% \mathrm{w} / \mathrm{v}$. Each cell containing hydrogel was cultured for 21 days by refreshing the culture medium each two days. At scheduled times, the metabolic activity of the incorporated chondrocytes was evaluated by MTS assay by using supplier instructions. The morphology of the incorporated chondrocytes was analysed by scanning electron microscopy (SEM). For this study at scheduled times, samples were fixed with $10 \% \mathrm{v} / \mathrm{v}$ formaldehyde, rinsed with water and dehydrated with ethanol series $(30 \%, 50 \%, 70 \%, 90 \% \mathrm{v} / \mathrm{v}$ and pure ethanol), then treated 
with hexamethyldisilazane and dried in a flow hood. Dried hydrogels were freeze fractured, situated on the stubs by using an appropriate adhesive tape, gold coated (thickness of gold coating between of about $20 \mathrm{~nm}$ ) and analysed by SEM in order to observe cells inside scaffolds. Each experiment was performed in triplicate.

\subsection{Histological analysis}

Hydrogels were fixed by submersion in a solution of formalin in DPBS pH 7.4 at a concentration of $10 \% \mathrm{v} / \mathrm{v}$ and then paraffin embedded. Subsequently, $4 \mu \mathrm{m}$ thick sections were cut, deparaffinized, rehydrated and evaluated with hematoxylin and eosin staining. The sections were analysed with optical microscope.

\subsection{Atomic force microscopy (AFM) analysis}

The experiment was performed at room temperature by keeping hydrogels in DPBS $\mathrm{pH}$ 7.4. For each sample, measurements were taken in three different regions; loading and unloading curves were obtained with a speed of $0.1 \mu \mathrm{m} \mathrm{s}^{-1}$. To determine the elastic modulus from the force/separation curves, the Hertz model has been used, considering, for all the obtained curves, force boundary between 10 and $40 \%$.

\subsection{Statistical analysis}

Data are presented as means \pm standard deviation, SD. T-test was employed for the statistical analysis, $p$ values littler than 0.05 were considered as statistically significant.

\section{Results}

\subsection{Synthesis of HA-EDA, HA-EDA- $g$ - $\alpha$-elastin and PHEA-DV}

Following a procedure patented by our research group, primary hydroxyl groups of HA have been derivatized with ethylenediamine (EDA) ${ }^{30}$ then the obtained HA-EDA derivative has been employed for the reaction with $\alpha$-elastin. The chemical characterization of both HA-EDA and HA-EDA- $g$ - $\alpha$-elastin derivatives has been already reported in previous articles. ${ }^{15,23}$ PHEA-DV has been produced following a synthetic procedure reported in Fig. 1A. ${ }^{1} \mathrm{H}-\mathrm{NMR}$ spectrum for the obtained product shows two typical peaks relative to the proton belonging to the doublebond protons of the DV moieties. The degree of derivatization (DD\%) in DV has been determined by comparing the peak integrals at $\delta 6.43$ and 6.90 relative to DV double-bond protons, with the peaks at $\delta 2.7,3.2$ and 3.6 of PHEA protons (Fig. 1B). The value of DD\% in DV was $5 \pm 2 \mathrm{~mol} \%$.

The molecular weight of PHEA-DV was $48 \mathrm{kDa}$ and the polydispersity index was 1.99 .

\subsection{Hydrogel production, swelling and degradation studies}

The crosslinking reaction via Michael-type addition, between free amino groups of HA-EDA or HA-EDA- $g$ - $\alpha$-elastin and vinyl portions of PHEA-DV, performed in DMEM in the absence of other supplement, takes place, for both derivatives, in 20 minutes at $37{ }^{\circ} \mathrm{C}$ (in the Fig. 2 it is reported as an example the crosslinking process between HA-EDA- $g$ - $\alpha$-elastin and PHEA-DV).

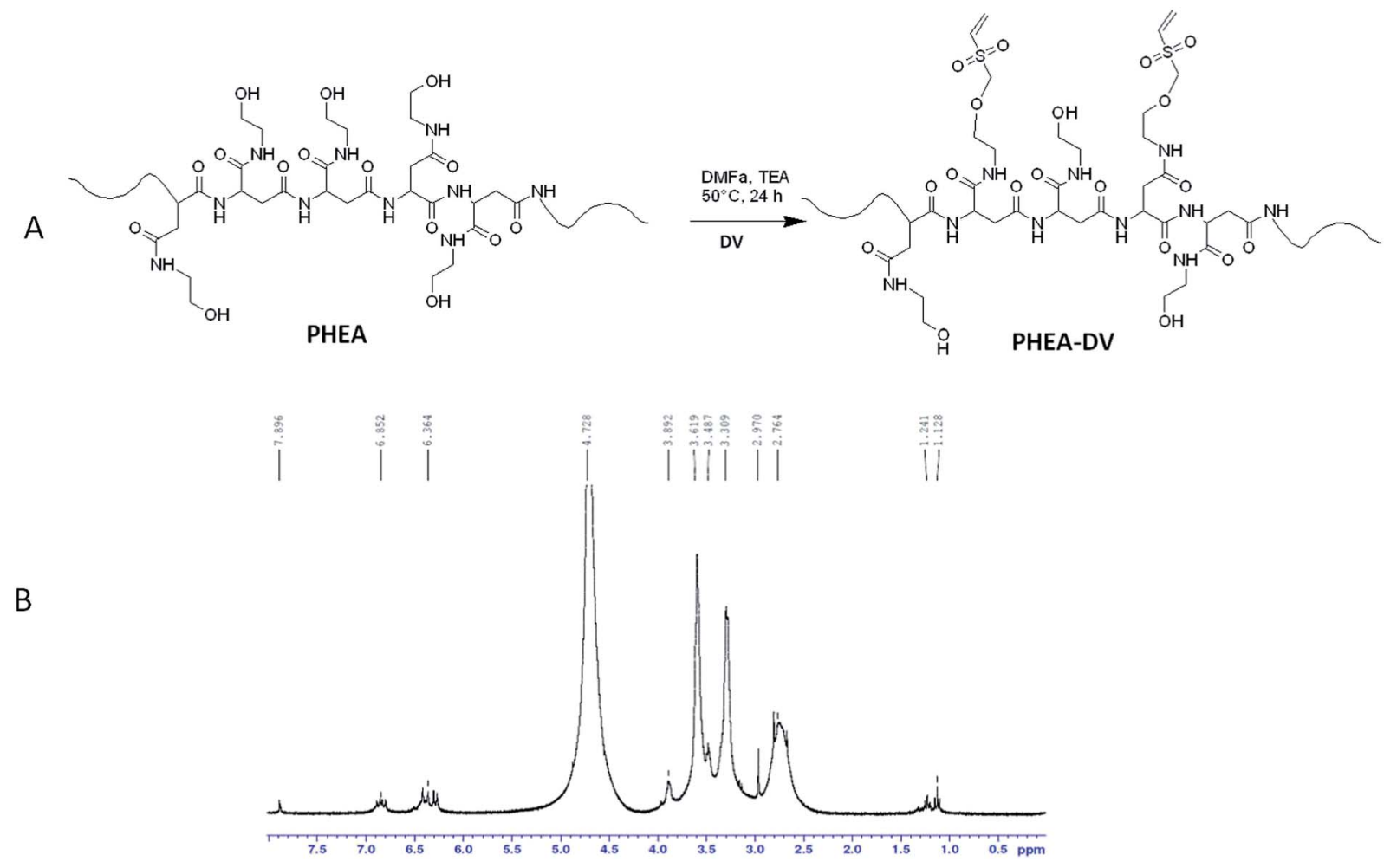

Fig. 1 (A) PHEA-DV synthesis from PHEA; (B) ${ }^{1} \mathrm{H}-\mathrm{NMR}$ spectrum of PHEA-DV. 


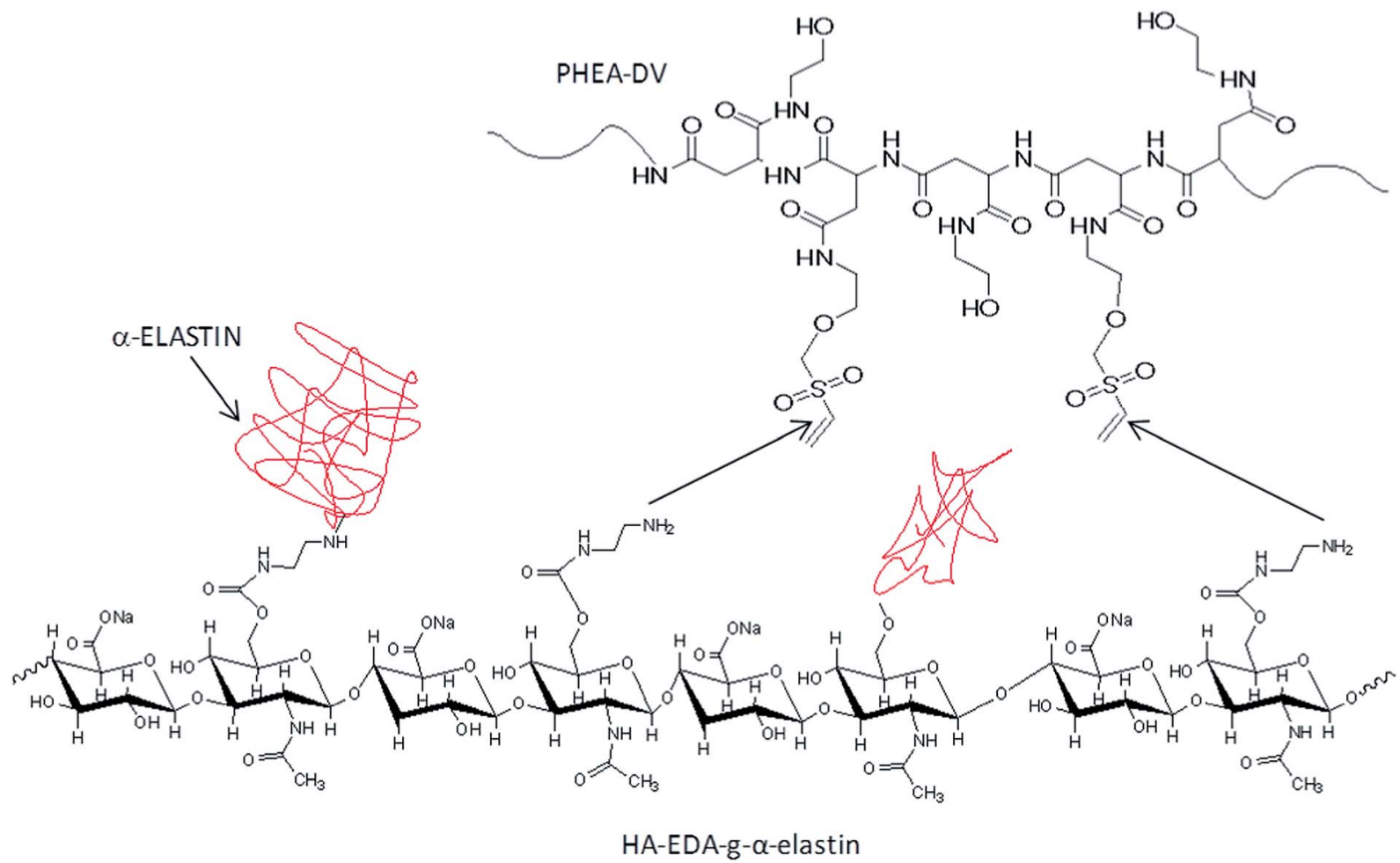

Fig. 2 Crosslinking process between HA-EDA- $g$ - $\alpha$-elastin and PHEA-DV.

HA-EDA/PHEA-DV and HA-EDA- $g$ - $\alpha$-elastin/PHEA-DV hydrogels do not show macroscopic shrinking process after 24 hours from crosslinking at $37{ }^{\circ} \mathrm{C}$. Obtained samples have been weighed and submerged in DBBS $\mathrm{pH} 7.4$ to evaluate their capacity to incorporate the aqueous buffer and to swell. Swelling behavior has been evaluated for 30 days and the trend is shown in Fig. 3.

It is possible to observe that both hydrogels swell with a similar profile after the first 2 weeks of incubation, indeed, after 24 hours, they increase their weight of $15 \%$ about $(14.60 \pm$ $4.18 \%$ for HA-EDA- $g$ - $\alpha$-elastin/PHEA-DV and $16.53 \pm 5.59 \%$ for HA-EDA/PHEA-DV) and they maintain this value (as well as the difference between the samples) basically unchanged until day 14. After 21 and 30 days of incubation, again no significant change in the swelling values is observed for HA-EDA- $g$ - $\alpha$ -

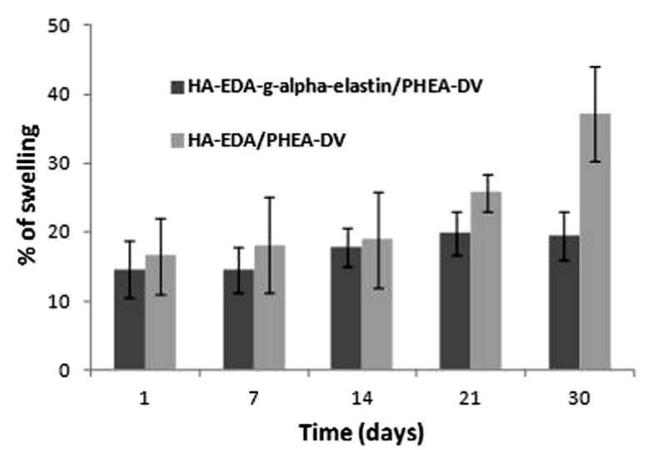

Fig. 3 Profiles of swelling\% over time for HA-EDA- $g$ - $\alpha$-elastin/PHEADV and HA-EDA/PHEA-DV hydrogels in DPBS $\mathrm{pH} 7.4,37^{\circ} \mathrm{C}$.
elastin/PHEA-DV hydrogels while it is possible to observe an increase in the swelling of HA-EDA/PHEA-DV hydrogel that reaches values of $25.73 \pm 2.72 \%$ and $37.17 \pm 6.82 \%$ at day 21 and 30 , respectively $(p=0.017)$. For degradation studies, the loss on weight of hydrogels was investigated until 30 days for the hydrolytic degradation, and until 72 hours for the enzymatic one. In Fig. 4 the hydrolytic (A) and enzymatic (B) degradation profile are shown.

Both HA-EDA- $g$ - $\alpha$-elastin/PHEA-DV and HA-EDA/PHEA-DV hydrogels degrade slowly in DPBS pH 7.4, indeed the recovered weight $\%$ after 30 days of incubation is $88.73 \pm 0.8 \%$ and $81.90 \pm 8.5 \%$, respectively (Fig. $4 \mathrm{~A}$ ). The degradation is increased in the presence of HAase; after 72 hours the recovered weight is $86.38 \pm 2.32 \%$ and $74.56 \pm 3.49$ for HA-EDA- $g$ - $\alpha$ elastin/PHEA-DV and HA-EDA/PHEA-DV hydrogels, respectively (Fig. 4B).

\subsection{Incorporation, metabolic activity evaluation and morphological analysis of bovine articular chondrocytes}

To incorporate bovine articular chondrocytes into the hydrogels, cells have been suspended in HA-EDA- $g$ - $\alpha$-elastin or HAEDA solution in DMEM before to add PHEA-DV, then the crosslinking reaction takes place in the presence of cells at $37^{\circ} \mathrm{C}$. The presence of cells does not influence the gelation time that remains, in both cases, equal to 20 minutes. The metabolic activity of incorporated cells has been evaluated by MTS assay and results have been reported in Fig. 5 .

The metabolic activity of chondrocytes incorporated in HAEDA- $g$ - $\alpha$-elastin/PHEA-DV hydrogels for all the incubation times 

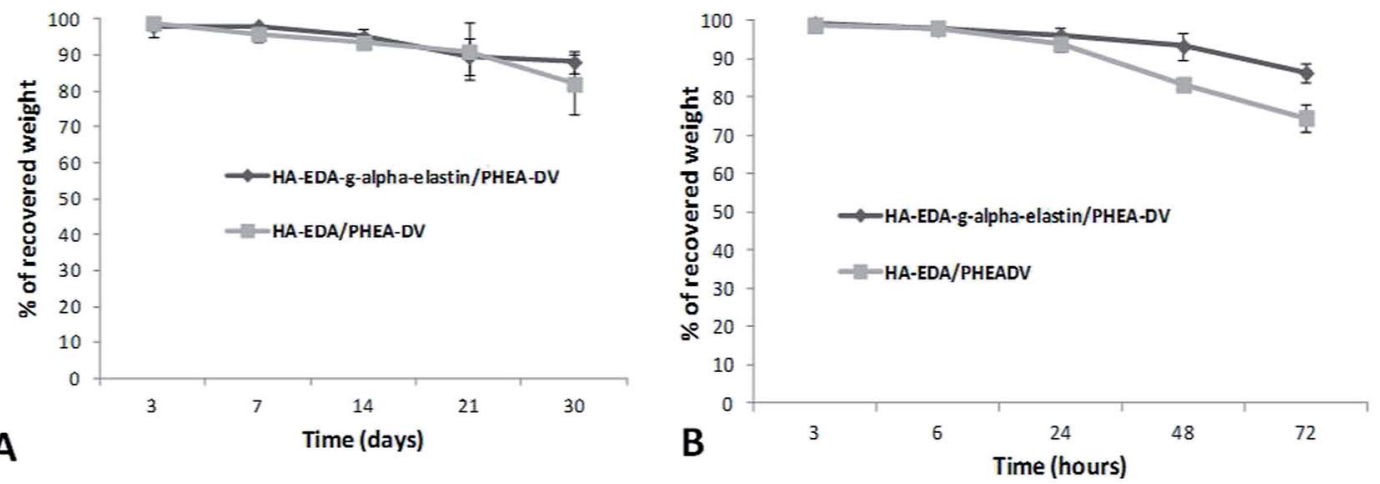

Fig. 4 Profiles of degradation, expressed as \% of recovered weight over time, for HA-EDA-g- $\alpha$-elastin/PHEA-DV and HA-EDA/PHEA-DV hydrogels in DPBS $\mathrm{pH} 7.4,37^{\circ} \mathrm{C}(\mathrm{A})$ and in DPBS $\mathrm{pH} 7.4,37^{\circ} \mathrm{C}$ in the presence of HAase (B).

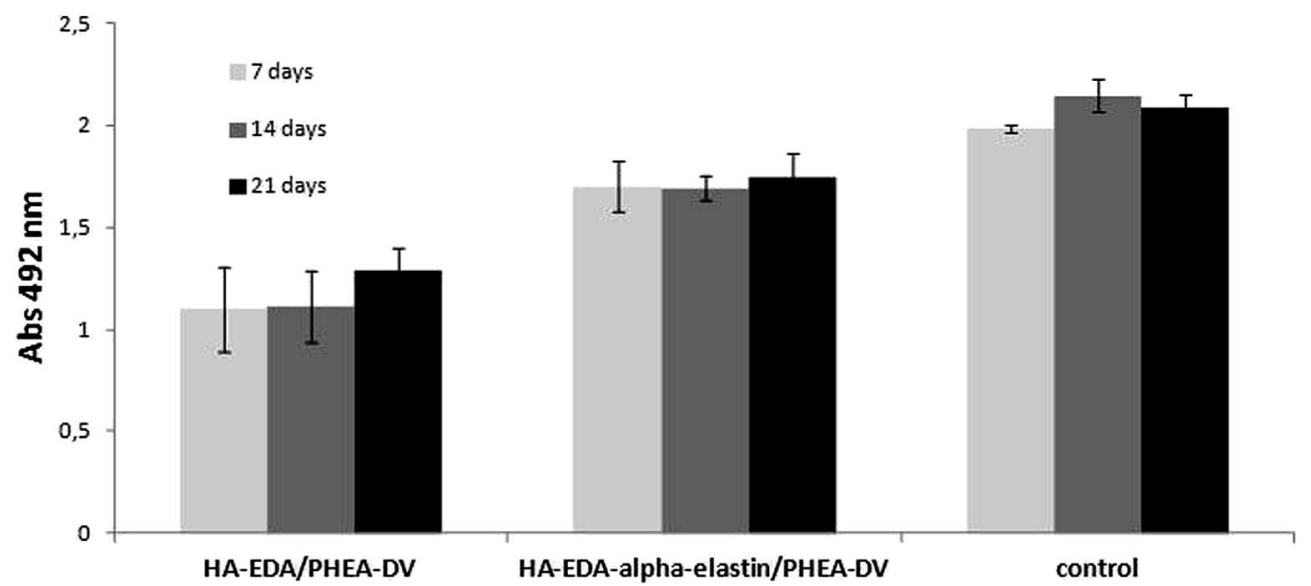

Fig. 5 MTS assay on chondrocytes encapsulated in HA-EDA- $g$ - $\alpha$-elastin/PHEA-DV and HA-EDA/PHEA-DV hydrogels. Data have been expressed as absorbance at $492 \mathrm{~nm}$ at 7,14 and 21 days of culture.

is about $80 \%$ of the control one (chondrocytes cultured in bidimensional condition) in particular it is $85.6 \pm 7.15 \%$ for day $7,78.8 \pm 1.86 \%$ for day 14 and $83.86 \pm 5.16 \%$ for day 21 . Cells incorporated in HA-EDA/PHEA-DV hydrogels show, for all the investigated times, a lower metabolic activity than that of the elastin containing samples. Compared to the positive control, the values are $55.25 \pm 10.48 \%$ for day $7,51.72 \pm 7.31 \%$ for day 14 and $62 \pm 3.78 \%$ for day 21 of incubation. Incorporated cells

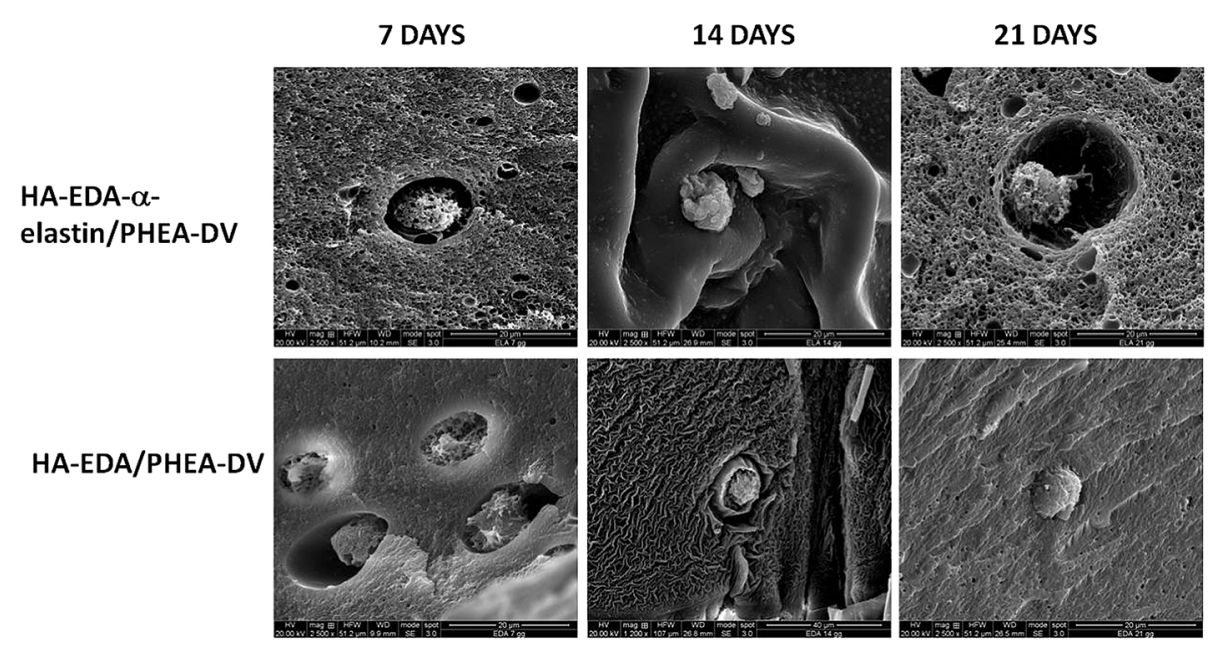

Fig. 6 SEM images of chondrocytes encapsulated in HA-EDA-g- $\alpha$-elastin/PHEA-DV and HA-EDA/PHEA-DV hydrogels after 7, 14 and 21 days of culture. 


\section{HA-EDA- $\alpha$-elastin/PHEA-DV}

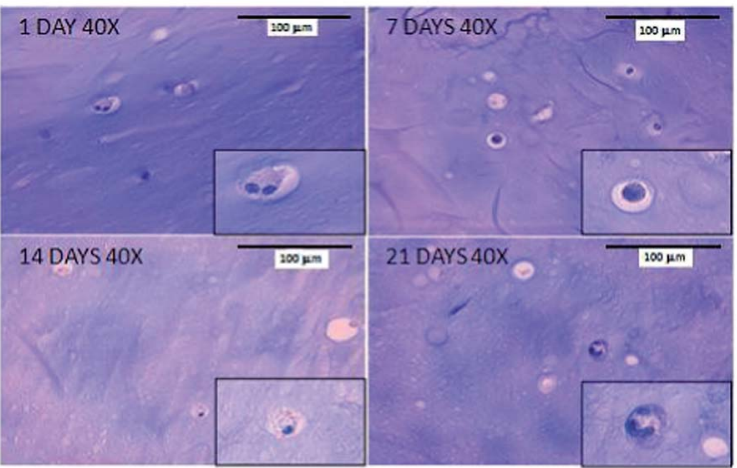

HA-EDA/PHEA-DV

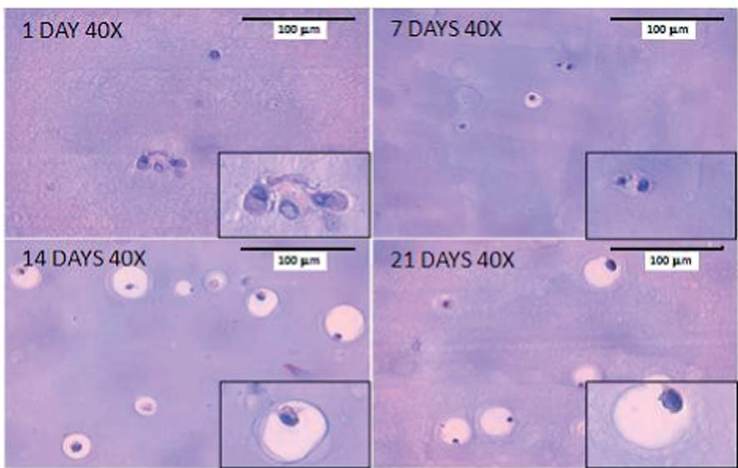

Fig. 7 Hematoxylin and eosin stained histological sections of HAEDA- $g$ - $\alpha$-elastin/PHEA-DV and HA-EDA/PHEA-DV cell containing hydrogels after 7,14 and 21 days of culture.

show in both HA-EDA- $g$ - $\alpha$-elastin/PHEA-DV and HA-EDA/PHEADV hydrogels, a typical round shape morphology how it is possible to see from the SEM images in Fig. 6. In all the cases, chondrocytes are close to each other but not organized in clusters.

Histological analysis confirm these results and show how chondrocytes are homogeneously distributed into the hydrogels (Fig. 7). However, no particular differences in the cell morphology are observed for elastin containing and elastin free hydrogels to support the different metabolic activity in the samples (see magnification images in the figures).

\subsection{Atomic force microscopy (AFM) analysis}

The effect of the presence of cells on the Young's modulus of the hydrogels has been investigated through AFM by analyzing cell free and cell containing samples cultured in complete DMEM for 48 hours before the experiment. Fig. 8 shows the values of Young's modulus for the investigated samples.

For cell containing hydrogels, HA-EDA- $g$ - $\alpha$-elastin/PHEA-DV sample shows a Young's modulus lower than that of HA-EDA/ PHEA-DV sample. However, interestingly the presence of cells greatly increases the elastic modulus of the elastin containing hydrogel from 0.096 to $0.710 \mathrm{MPa}$. This effect was not observed for the hydrogel without elastin which shows, in the presence of chondrocytes, only a slight and not significant increase from 0.170 to $0.191 \mathrm{kPa}$. To confirm that the increase in the Young's

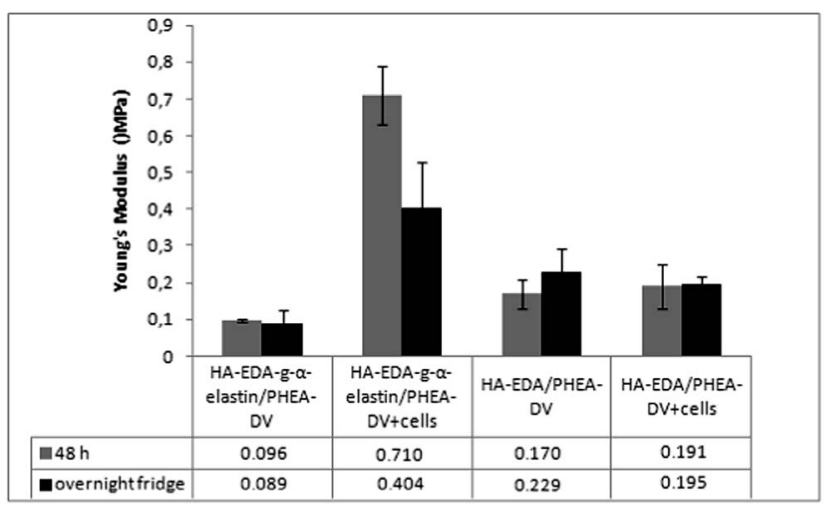

Fig. 8 Young's modulus of cell containing and cell free HA-EDA- $g-\alpha-$ elastin/PHEA-DV and HA-EDA/PHEA-DV hydrogels. Blacks histograms refer to the same samples kept for one night in the fridge.

modulus for HA-EDA- $g$ - $\alpha$-elastin/PHEA-DV sample was due to the presence of viable cells, samples were kept in the refrigerator overnight $\left(4^{\circ} \mathrm{C}\right)$ to cause cell death prior to repeat the AFM measurements. The cell death was confirmed by performing the MTS test on samples incubated overnight in the refrigerator (data not shown). Referring to the values obtained after 48 hours of incubation, no significant change was observed for cell free hydrogels as well as for cell containing HA-EDA/PHEA-DV sample. On the contrary the elastic modulus of cell containing HA-EDA- $g$ - $\alpha$-elastin/PHEA-DV sample decreases significantly from 0.710 to $0.404 \mathrm{MPa}$ after cell dying.

\section{Discussions}

The target pursued in this work has been the development of an in situ forming hydrogel for the potential treatment of articular cartilage defects. Hyaluronic acid derivatives bearing free amino pendant groups (HA-EDA and HA-EDA- $g$ - $\alpha$-elastin) have been crosslinked via Michael-type reaction in aqueous medium and mild conditions with PHEA-DV, a synthetic and water soluble polymer containing vinyl sulfone groups (Fig. 2). The time occurring for the crosslinking is very important for an optimal performance of an in situ forming hydrogel during in vivo administration. Indeed, if the reaction takes place too quickly, gelation could occur into the syringe and the surgeon will not have the time necessary to inject the polymer dispersion into the articulation. On the other hand if the crosslinking reaction is too slow, the solution could leak out of the cartilage defect once injected. HA-EDA/PHEA-DV and HA-EDA$g$-1 $\alpha$-elastin/PHEA-DV form stable chemical hydrogels after 20 minutes of incubation in phosphate buffer $\mathrm{pH} 7.4$ at $37^{\circ} \mathrm{C}$ both in presence or not of bovine articular chondrocytes. In our opinion this time interval could potentially allow the loading of the arthroscopic syringe, to proceed without undue haste and to obtain an optimal in situ gelation. Prepared hydrogels are able to incorporate phosphate buffer and to reach a swelling equilibrium within 24 hours of incubation. The ability to swell in aqueous medium is a crucial parameter for a scaffold since it can influence the amount of oxygen and nutrients into the construct and consequently the viability of 
the incorporated cells. Moreover, together with the rate of degradation, it can also affect neocartilage formation by chondrocytes in vivo as reported in the literature. ${ }^{31}$ However if an excessive swelling of the hydrogel occurs, the size of the construct could change drastically and affect its ability to stay in place into the cartilage defect after the in vivo implantation. The volume increase in HA-EDA- $g$ - $\alpha$-elastin/PHEA-DV hydrogels is not considerable and it remains constant during the days of analysis. Instead, HA-EDA/PHEA-DV hydrogel increases its volume after 21 days of incubation as well as its weight because of an increased amount of absorbed water (Fig. 3), probably due the occurrence of a degradation process. The different behavior between investigated hydrogels could be attributable to the presence of $\alpha$-elastin which could reduce the water uptake because of its hydrophobic domains. The degradation profile of elastin containing and elastin free hydrogels in the presence or not of hyaluronidase seems to be optimal to allow the maintenance of their structural integrity and thus their function as cell scaffolds for the time necessary for producing new extracellular matrix (Fig. 4). On the other hand, the sensitivity to enzymatic degradation suggests that both HA-EDA/PHEA-DV and HA-EDA- $g$ - $\alpha$-elastin/PHEA-DV hydrogels are potentially biodegradable and they do not remain in the implant site for long times. To solve the problems related to the incorporation of cells into an in situ forming system, it is necessary to answer to the following questions: (1) is it possible to obtain a homogeneous distribution of the cells in the system? (2) Is the system able to promote the exchange of nutrients and oxygen, and then to support cell viability? (3) Incorporated cells actually take contact with the matrix recognizing it as a substrate? First, cell distribution into a hydrogel is related to the viscosity of the gel forming solution. Both HA-EDA and HA-EDA- $g$ - $\alpha$-elastin solution in DMEM at $3 \% \mathrm{w} / \mathrm{v}$ can be easily mixed with the cell suspension simply by pipetting avoiding to employ more energetic mixing procedure (for example vortex) that can damage the cells. The confirmation of the uniform cell distribution in the produced hydrogels has been obtained by histological studies. The MTS test allows to determine not only the viability of the incorporated cells but also to understand their state of being through the evaluation of their metabolic activity. If a reduction in the metabolic activity is obtained (compared to a positive control) this result could indicate that part of the cells are dead or even if alive, they are not healthy. The metabolic activity of the chondrocytes into HA-EDA- $g$ - $\alpha$ elastin/PHEA-DV hydrogel is only slightly lower than that of the cells cultured in 2D conditions but it is significantly greater than that of cells incorporated into the HA-EDA/PHEADV hydrogels (Fig. 5). Probably this result cannot be related to a different ability of HA-EDA/PHEA-DV and HA-EDA- $g$ - $\alpha$ elastin/PHEA-DV hydrogels in allowing the exchange of gases and liquids since, as already shown and discussed, the degree of swelling is similar for both investigated samples, especially in the early days of analysis. It is reasonable to believe that the greater metabolic activity observed for cells into HA-EDA- $g-\alpha-$ elastin/PHEA-DV hydrogel can be related to the presence of elastin. Indeed, as demonstrated in our previous work, ${ }^{23}$ elastin contains adhesion domains that, in this case, could allow the recognition of the material by chondrocytes, as well the formation of a microenvironment similar to that of the native extracellular matrix. With this in mind, we have tried to functionalize HA with high amount of elastin starting from a HA-EDA with a high degree of functionalization in aminic portions. In any case, SEM analysis (see Fig. 6) confirms that in both HA-EDA- $g$ - $\alpha$-elastin/PHEA-DV and HA-EDA/PHEA-DV hydrogels, chondrocytes show their typical round shape, thus suggesting that cells maintain their corrected phenotype. The elastic modulus of both HA-EDA- $g$ - $\alpha$-elastin/PHEA-DV and HA-EDA/PHEA-DV hydrogels, is higher than that reported in the literature and obtained through AFM analysis on hydrogels produced with various natural and synthetic polymers. ${ }^{32}$ Viable cells in the HA-EDA/PHEA-DV hydrogel determine only a slight increase in the elastic modulus, whereas their presence increases greatly (about ten fold) the elastic modulus of HA-EDA- $g$ - $\alpha$-elastin/PHEA-DV hydrogel. This result seems to confirm the hypothesis of the recognition of chondrocytes of the adhesion moieties on elastin. Indeed, it is reasonable to think that when cells establish specific interactions with the material they can compact the hydrogel itself by acting as biological crosslinkers, thus causing a pronounced increase in elastic modulus. To confirm that the increase in the elastic modulus in the elastin containing hydrogel is correlated to cells viability, samples have been placed in the fridge overnight, in order to cause cell death. As we expected, the death of the cells determines a decrease in the Young's modulus only in HA-EDA- $g$ - $\alpha$-elastin/PHEA-DV hydrogel.

\section{Conclusions}

Two different HA based in situ forming hydrogels have been obtained by the crosslinking reaction between the polysaccharide derivatives (named HA-EDA and HA-EDA- $g$ - $\alpha$-elastin) and the divinylsulfone functionalized $\alpha, \beta$-poly $(N-2$-hydroxyethyl)-DL-aspartamide (named PHEA-DV). The gelling reaction takes place without any catalyst and in Dulbeccos Modified Eagles Medium (DMEM) giving rise, in about 20 minutes, to the production of chemical hydrogels. The presence of $\alpha$-elastin makes hydrogels able to maintain a swelling equilibrium until 30 days of incubation in phosphate buffer without drastic changes in the volume of samples. HA-EDA/PHEA-DV and HAEDA- $g$ - $\alpha$-elastin/PHEA-DV hydrogels show a good resistance towards hydrolytic and enzymatic degradation. It has been demonstrated that it is possible to incorporate during the hydrogel formation viable articular chondrocytes that remain metabolically active until 21 days of incubation showing a typical round shape similar to that in the native tissue. Both metabolic assay and AFM mechanical test seem to demonstrate that cells recognize $\alpha$-elastin in the scaffold through specific interactions that favor a better metabolic activity and allow the improvement of the elastic modulus of the hydrogel. To the light of the results we believe that HA-EDA- $g$ - $\alpha$-elastin/PHEA-DV in situ forming hydrogel could represent an optimal candidate for cartilage tissue engineering purposes. 


\section{References}

1 A. Wolf and B. Pfleger, Bull. W. H. O., 2003, 81, 646-656.

2 B. Balakrishnan, N. Joshi, A. Jayakrishnan and R. Banerjee, Acta Biomater., 2014, 10, 3650-3663.

3 M. Brittberg, A. Lindahl, A. Nilsson, C. Ohlsson, O. Isaksson and L. Peterson, N. Engl. J. Med., 1994, 331, 889-895.

4 D. Nesic, R. Whiteside, M. Brittberg, D. Wendt, I. Martin and P. Mainil-Varlet, Adv. Drug Delivery Rev., 2006, 58, 300-322.

5 E. Hunziker, Osteoarthritis Cartilage, 2002, 10, 432-463.

6 S. Redman, S. Oldfield and C. Archer, Eur. Cells Mater., 2005, 9, 23-32.

7 E. Hunziker, Clin. Orthop. Relat. Res., 1999, 367, 135-146.

8 L. Lu, X. Zhu, R. Valenzuela, B. Currier and M. Yaszemski, Clin. Orthop. Relat. Res., 2001, 391, 251-270.

9 C. Chung and J. A. Burdick, Adv. Drug Delivery Rev., 2008, 60, 243-262.

10 S. H. M. Ś'ontjens, D. L. Nettles, M. A. Carnahan, L. A. Setton and M. W. Grinstaff, Biomacromolecules, 2006, 7, 310-316.

11 D. Wang, S. Varghese, B. Sharma, I. Strehin, S. Fermanian, J. Gorham, D. Fairbrother, B. Cascio and J. Elisseeff, Nat. Mater., 2007, 6, 385-392.

12 H. Tan, C. R. Chu, K. A. Payne and K. G. Marra, Biomaterials, 2009, 30, 2499-2506.

13 M. Kim, Y. Shin, B. Hong, Y. J. Kim, J. S. Chun, G. Tae and Y. H. Kim, Tissue Eng., Part C, 2010, 16, 1-10.

14 Y. Park, M. P. Lutolf, J. A. Hubbell, E. B. Hunziker and M. Wong, Tissue Eng., Part C, 2004, 10, 515-522.

15 F. S. Palumbo, G. Pitarresi, C. Fiorica, P. Matricardi, A. Albanese and G. Giammona, Soft Matter, 2012, 8, 49184927.

16 R. Jin, L. S. M. Teixeira, P. J. Dijkstra, C. A. van Blitterswijk, M. Karperien and J. Feijen, J. Controlled Release, 2011, 152, 186-195.

17 F. S. Palumbo, M. Di Stefano, A. Palumbo Piccionello, C. Fiorica, G. Pitarresi, I. Pibiri, S. Buscemi and G. Giammona, RSC Adv., 2014, 4, 22894-22901.
18 C. Civiale, M. Licciardi, G. Cavallaro, G. Giammona and M. Mazzone, Int. J. Pharm., 2009, 378, 177-186.

19 M. Licciardi, G. Cavallaro, M. Di Stefano, C. Fiorica and G. Giammona, Macromol. Biosci., 2011, 11, 445-454.

20 G. Pitarresi, M. A. Casadei, D. Mandracchia, P. Paolicelli, F. S. Palumbo and G. Giammona, J. Controlled Release, 2007, 119, 328-338.

21 G. Pitarresi, C. Fiorica, F. S. Palumbo, F. Calascibetta and G. Giammona, J. Biomed. Mater. Res., Part A, 2012, 100, 1565-1572.

22 G. Pitarresi, C. Fiorica, F. S. Palumbo, S. Rigogliuso, G. Ghersi and G. Giammona, J. Biomed. Mater. Res., Part A, 2014, 102, 1334-1341.

23 F. S. Palumbo, G. Pitarresi, C. Fiorica, S. Rigogliuso, G. Ghersi and G. Giammona, Mater. Sci. Eng. C, 2013, 33, 2541-2549.

24 R. Mendichi, A. G. Schieroni, G. Cavallaro, M. Licciardi and G. Giammona, Polymer, 2003, 44, 4871-4879.

25 X. Z. Shu, Y. Liu, Y. Luo, M. C. Roberts and G. D. Prestwich, Biomacromolecules, 2002, 3, 1304-1311.

26 F. S. Palumbo, G. Pitarresi, D. Mandracchia, G. Tripodo and G. Giammona, Carbohydr. Polym., 2006, 66, 379-385.

27 S. M. Partridge, H. F. Davis and G. S. Adair, Biochem. J., 1955, 61, 11-21.

28 B. Jeong, Y. H. Bae and S. W. Kim, Macromolecules, 1999, 32, 7064-7069.

29 C. Fiorica, G. Pitarresi, F. S. Palumbo, M. D. Stefano, F. Calascibetta and G. Giammona, Int. J. Pharm., 2013, 457, 150-157.

30 G. Giammona, F. Palumbo and G. Pitarresi, IWO Patent Application 061005A, 2010.

31 C. Chung, J. Mesa, M. A. Randolph, M. Yaremchuk and J. A. Burdick, J. Biomed. Mater. Res., Part A, 2006, 77, 518-525.

32 C. D. Markert, X. Guo, A. Skardal, Z. Wang, S. Bharadwaj, Y. Zhang, K. Boninb and M. Guthol, J. Mech. Behav. Biomed. Mater., 2013, 27, 115-127. 